\title{
Book review: Im Inneren der Bauverwaltung: Wirkmacht und Eigenlogik administrativer Praktiken in der Bauverwaltung
}

\author{
Lineo U. Devecchi ${ }^{1,2}$ \\ ${ }^{1}$ Universität Zürich, Institut für Politikwissenschaft, Affolternstrasse 56, 8050 Zürich, Switzerland \\ ${ }^{2}$ Ostschweizer Zentrum für Gemeinden, Fachhochschule St. Gallen, Rosenbergstrasse 59, 9001 St. Gallen, \\ Switzerland
}

Correspondence to: Lineo U. Devecchi (devecchi@ipz.uzh.ch)

Published: 23 March 2017

Schmidt, M.: Im Inneren der Bauverwaltung: Wirkmacht und Eigenlogik administrativer Praktiken in der Bauverwaltung, Bielefeld: transcript, 338 pp., ISBN: 978-3-8376-3333-7, EUR 39.99, 2016.

Welche Investorin, welcher Architekt, welche Bauherrin hat nicht schon Entscheidungen der kommunalen Bauverwaltungen in Frage gestellt, den Verwaltungsangestellten Unwissenheit und Unvermögen vorgeworfen, ihr Vorgehen und ihre Regeln als zu rigide bezeichnet, ihnen das Misslingen einer kühnen Bauidee angehaftet? Und welche ebendieser Personen hat in politischen Diskussionen nicht auch schon die Vorzüge des Schweizer Föderalismus, der starken Autonomie der lokalen politischen Ebene oder der kommunalen Demokratie angepriesen? Wir fragen uns zudem immer wieder auch leicht ironisch, was in den Verwaltungen unserer National- und Lokalstaaten so passiert, wie dort entschieden wird, wer dort, und wenn überhaupt, wie arbeitet. Im Spannungsfeld ebendieser Fragen kann der vorliegende Band verortet werden, mit dem sich Michaela Schmidt ins Innere der Bauverwaltung vorwagt, um der Eigenlogik und der Wirkmacht administrativer Praktiken bei Bauprojekten detailliert und analysierend auf den Grund zu gehen. Ich kann es vorweg nehmen: Der Anspruch, den Michaela Schmidt sich in ihrem Buch selbst setzt, ist ambitioniert. Ebenfalls vorweg nehmen kann ich, dass es ihr weitestgehend sehr überzeugend gelingt, dabei die Black Box der Schweizer Gemeinde zu öffnen, genau hinein zu schauen und spannende Erkenntnisse über ihr Funktionieren zu gewinnen.
Aber der Reihe nach! Der erste Aufhänger für Michaela Schmidts Vorhaben, das Innenleben von Bauverwaltungen unter die Lupe zu nehmen, ist das Aufweichen des Dualismus zwischen Stadt und Land, das in den letzten Jahrzehnten mit der Bildung von funktionalen Agglomerationsräumen zugenommen hat. Der zweite Orientierungspunkt zum Start der vorliegenden Studie ist den Autoren des ,städtebaulichen Portraits“ (Diener et al., 2006) zu verdanken, die die Schweizer Gemeinde vor einer Dekade aufgrund ihrer Autonomie als „Antiurbanitätsmolekül“ bezeichnet haben. Schmidt will im Inneren der Bauverwaltungen mittelgrosser Gemeinden nach Ursachen und Antworten bezüglich dieser These suchen. Dabei will sie aufzeigen, wie eine Repositionierung der Gemeinden als aktivere Gestalterinnen der immer urbaner werdenden Schweizer Siedlungslandschaft aussehen könnte. Der dritte Aufhänger für den vorliegenden Band - der Schmidts Forschungstätigkeiten im Rahmen ihrer Dissertation an der ETH Zürich abbildet - ist das aus der Sicht der Autorin bislang unzulängliche wissenschaftliche Befassen mit der (Schweizer) Gemeinde. Ziel ihres hier präsentierten Forschungsvorhabens ist es demnach auch, das Funktionieren von Gemeindeverwaltungen mit innovativen methodischen Ansätzen aufzuzeigen und zu neuen theoretischen Erkenntnissen zu verarbeiten.

Konkret fragt die Autorin erstens nach den administrativen Praktiken und Prozessen beim Begleiten, Beurteilen und Bewilligen von Bauprojekten auf der kommunalen Ebene. Zweitens fokussiert sie sich auf die Veränderungen, die einem Bauprojekt nach der Eingabe widerfahren und nimmt dabei drittens den Einfluss der Verwaltung in den Blick, den sie auf unsere gebaute Umwelt haben kann - respektive ha- 
ben könnte. Viertens fragt sie danach, ob bisherige Konzepte der Verwaltungswissenschaften und Organisationssoziologie zu kurz greifen, um die Wirkmacht und die Eigenlogik von Verwaltungen aufzuzeigen. Die Autorin nimmt sich im vorliegenden Band auf 314 Textseiten und mittels zahlreichen illustrativen Grafiken und Abbildungen diesen Fragen an. Sie nimmt die Leserschaft mit auf eine faszinierende Reise ins Innere der Bauverwaltung, zeigt die ersten Kontakte zwischen Bauwilligen und Verwaltung auf, bringt uns - immer auf den Spuren von Bauprojekten - den verschlungenen administrativen Pfaden zwischen verschiedenen Büros und Sitzungsräumen näher und lässt an Entscheidungen teilhaben, zu denen auch viele Beteiligte am Baubewilligungsverfahren wie Akteurinnen und Akteure aus Architektur, Bauherrenoder Investorenschaft, nur selten Zugang haben.

In den ersten beiden Kapiteln führt Michaela Schmidt in ihren Forschungsgegenstand ein, klärt die genauen Forschungsfragen, die Fallauswahl sowie ihr qualitativethnographisches Vorgehen - neben teilnehmendem Beobachten und mittels Interviews ging die Autorin auch videoethnographisch vor - und führt interdisziplinär in den Stand der Forschung ein. Im dritten Kapitel, das als Bindeglied zwischen Theorie, Methode und Empirie fungiert, stellt die Autorin die drei Bauverwaltungen in Visp, Wetzikon und St. Margrethen vor. Alle drei Gemeinden stehen unter ökonomischem Druck - die ersten beiden wachsen stark, die dritte verlor einen Grossteil der lokalen Arbeitsplätze aufgrund struktureller ökonomischer Veränderungen. Dies stellt die raumordnerischen Tätigkeiten vor neue Herausforderungen. Erstmals mit ins Innere der Bauverwaltung nimmt uns die Autorin dann im vierten Kapitel. Sie beschreibt die Wege, die Bauwillige von der Eingangstür zum Schalter der Bauabteilung zurücklegen müssen anschaulich als durch die Arbeitsprozesse und Machtstrukturen der kommunalen Verwaltung organisiert. So sind die leeren Flure nicht nur Zeichen des effizienten administrativen Arbeitens, sondern aus der Sicht externer „Bittstellerinnen und Bittstellern“ auch die bauliche Manifestierung von Zuständigkeiten und Hierarchie. Denn hinter den geschlossenen Türen sind die „Eingeweihten" ihrerseits durch Türdurchbrüche und für die Öffentlichkeit verborgene Treppenhäuser miteinander verbunden und in ständiger Kommunikation, um das Baubewilligungsverfahren - das zentrale ordnende Element im Bauprozess - möglichst effizient zu administrieren.

Den verschiedenen administrativen Schritten im Baubewilligungsverfahren begegnen wir in den darauffolgenden Kapiteln fünf bis neun immer wieder. Dabei widmet die Autorin den wichtigsten Meilensteinen in der administrativen Karriere eines Bauprojekts je ein einzelnes Kapitel, worin sie die Logik und Wirkmöglichkeiten der Bauverwaltungen detailliert und einleuchtend beschreibt. Schmidt zeigt anhand der administrativen Praktiken des Ordnens und Verwaltens, mittels Darstellungen von Sprechstunden, Stadtbildund Baukommissionssitzungen die Rolle des Baubewilligungsverfahrens - dem ,administrativen Schrittmacher“ der
Bauverwaltung - auf. Dabei ist der Baubewilligungsentscheid der Fokalpunkt sowohl der Bauwilligen, da mitunter ihre wirtschaftliche Existenz von einem positiven Entscheid abhängt, als auch der Bauverwaltungen, deren Job grundsätzlich die möglichst effiziente Organisation des Bewilligungsprozesses darstellt. Es zeigt sich jedoch, dass die in der Theorie - in den juristischen Regularien, ebenso wie in den heutigen Lehrbüchern der Planung - klar strukturierten Bewilligungsabläufe, in der administrativen Realität deutlich flexibler zu sein scheinen. Die Bauverwaltung hat Wirkmacht, weil sie beispielsweise Projekte, die für das wirtschaftliche Überleben des grössten ansässigen Industriebetriebes entscheidend sind, beschleunigen kann. Dies resultiert in einer prominenteren Ablageposition der Akte im Aktenschrank, was eine frühere administrative Bearbeitung bedeutet. Die Verwaltung kann Bewilligungsprozesse auch verlangsamen und dabei umfassendere Auseinandersetzungen mit einem geplanten, aber kritisch betrachteten Projekt ermöglichen oder bei Bedarf erzwingen - zum Beispiel mittels der zusätzlich angeordneten Diskussion des Projektes in der Stadtbildkommission, in denen die eigentlich rein baujuristische administrative Beurteilung zugunsten einer qualitativeren Aushandlung in der grösseren Gruppe aufgeweicht wird. Die Bauverwaltung kann also die Art und Weise verändern, wie intensiv und wie breit abgestützt am konkreten Bauprojekt gearbeitet wird, um es schliesslich zur Bewilligungsreife zu bringen. Die von Schmidt in diesem Zusammenhang beschriebene ,administrative Zeitrechnung“, in die ein Bauprojekt mit der Eingabe eintritt, die neben der geradlinigen und effizienten Bewilligung eben auch die kurvige, und nicht immer vorwärtsgerichtete „Zeitschlange“ kennt, beschreibt diese vielfältige administrative Logik Schweizer Gemeinden einleuchtend.

Im neunten Kapitel präsentiert Michaela Schmidt ihre Resultate in einer Schlussbetrachtung. Sie zeigt dabei nochmals auf, wie das Baubewilligungsverfahren als „Prüfapparat" funktioniert, in dem bestimmte Schritte wie die Baubewilligungsentscheidung als „Nadelöhr“ zwingend zu passieren sind, in dem aber auch immer wieder kreative Momente des administrativen Eingreifens möglich sind. Beide Prozessarten wirken sich dabei dynamisch auf die Gestalt der Bauprojekte ein - und somit auf unsere Siedlungslandschaften. Schmidt plädiert für ein aktiveres und relationaleres Verständnis von Verwaltung: Verwalten bezieht sich demgemäss nicht nur auf das effiziente Organisieren der juristisch vorgegebenen Prozesse, sondern beinhaltet auch das kreative Umsetzen administrativer Wirkmöglichkeiten - angetrieben durch einzelne, durchaus eigenständig handelnde Personen. Diese Feststellung hat aus ihrer Sicht nicht nur Konsequenzen für ein künftiges Selbstbild in den Kommunalverwaltungen, sondern auch für weitere wissenschaftliche Analysen. Einerseits sollten sich kommunale Administrationen ihrer Wirkmacht noch stärker bewusst werden (Gemeinden sind also nie per se, können aber durchaus „Antiurbanitätsmoleküle" sein) und diese auch aktiver einsetzen. Andererseits ge- 
nügt es nach Schmidt wissenschaftlich nicht mehr, Verwaltungen lediglich als Reproduktionsstätten gesetzlicher Vorgaben zu analysieren.

Der vorliegende Band hält sein titelgebendes Versprechen, umfassend ins Innere der Bauverwaltungen zu blicken, vollständig. Insbesondere die empirischen Kapitel geben substantielle, analytisch stringente, spannende und teilweise auch sehr überraschende Einblicke in die administrativen Praktiken, die in Bau- und Siedlungsentwicklungsprozessen auch künftig unumgänglich bleiben werden - oder, um Schmidts Haltung zu explizieren, noch wichtiger werden sollten. Leider bleiben die konkreten inhaltlichen Auswirkungen der administrativen Wirkmacht auf konkrete Bauprojekte trotz - oder aufgrund? - der analytischen Dichte der einzelnen Diskussionsmomenten und Entscheidungssituationen, etwas verborgen. Hier wäre für die stärker am Bauprozess interessierte Leserschaft ein grösserer Fokus auf konkrete inhaltliche Veränderungen der Projekte angebracht gewesen - was zwar methodisch schwieriger gewesen wäre, aber eventuell auch die wissenschaftliche Nachvollziehbarkeit der Analyse und die Interpretation der Resultate noch verbessert hätte.

Den trotz diesem Einwand beispielhaft gelungenen empirischen Kapiteln gegenüber fällt die theoretische und methodische Einbettung Schmidts etwas ab. So lehnt sie sich zum Beispiel mit der Diagnose, dass die starre Gemeindedefinition aus bisherigen sozialwissenschaftlichen Studien das Erfassen und Analysieren aktiver Verwaltungen stark erschweren, etwas gar weit aus dem Fenster. Vor allem die Governance-Literatur (z.B. Stone, 1989; Pierre und Peters, 2000) und die Abhandlungen zur street level bureaucracy (z.B. Lipsky, 1980) fassen den Gemeinde- und Verwaltungsbegriff sehr breit, analysieren konkretes Akteurshandeln und individuelle Agency und lassen auch die konkreten Auswirkungen administrativer Tätigkeiten auf unsere Lebensrealitäten nicht aussen vor. Ebenfalls bleibt die Rolle der transsequentiellen Analyse (TSA) teilweise unklar. Ist sie nun Theorie, Methode oder Methodologie? Diese Unklarheiten könnten entweder dem etwas zu theoretisch abgehandelten Methodenbeschrieb geschuldet sein - was angesichts des breiten Fundus' an empirischem Material schade ist -, oder an der möglicherweise zu wenig von anderen ethnographischen Methoden abgrenzbaren TSA liegen.
Gleichwohl gelingt es Michaela Schmidt, einen äusserst wertvollen Beitrag zur Klärung dessen zu leisten, was innerhalb kommunaler Administrationen und spezifisch auf Bauverwaltungen vor sich geht, welche administrativen Ressourcen vorhanden sind und wie diese im Rahmen der kommunalen Autonomie genutzt werden (könnten). Dabei macht Schmidt auch aufgrund ihrer sprachlichen Kreativität - sie entwirft im Grunde im Laufe des Buches ein neues Vokabular für die Beschreibung der ,administrativen Maschine“ -, kommunale Verwaltungspraktiken intuitiver erlebbar. Das Buch ist somit all jenen Interessierten aus Wissenschaft und Praxis besonders zu empfehlen, die sich die von mir einleitend aufgeworfenen Fragen bereits gestellt haben - und das dürften einige sein!

\section{Literatur}

Diener, R., Herzog, J., Meili, M., und Schmidt, Ch.: Die Schweiz: Ein städtebauliches Portrait, Basel, Birkhäuser, 2006.

Lipsky, M.: Street-level Bureaucracy: Dilemmas of the Individual in Public Services, New York, Russel Sage Foundation, 1980.

Pierre, J. und Peters, B. G.: Governance, Politics and the State, London, Macmillan Press, 2000.

Stone, C. N.: Regime Politics: Governing Atlanta, 1946-1988, Lawrence, University Press of Kansas, 1989. 